\title{
Some new refinements of Heinz inequalities of matrices
}

Shaoheng Wang*

\section{"Correspondence:} wshcher@163.com

School of Mathematics and Statistics, Chongqing Three Gorges University, Wanzhou, 404100, China

\begin{abstract}
Firstly, a new refinement of the Heinz inequality is given. Then, by using the inequality, some new refinements of Heinz inequalities of matrices are given. Lastly, we declare that the results are better than the ones in (Kittaneh in Integral Equ. Oper. Theory 68:519-527, 2010), but we cannot say that our results are better than the ones in (Feng in J. Inequal. Appl. 2012:18, 2012, doi:10.1186/1029-242X-2012-18).
\end{abstract}

Keywords: new refinements; Heinz inequality; convex function; unitarily invariant norm

\section{Introduction}

For every unitarily invariant norm, we have the Heinz inequalities (see [1])

$$
2\left\|A^{\frac{1}{2}} X B^{\frac{1}{2}}\right\| \leq\left\|A^{v} X B^{1-v}+A^{1-v} X B^{v}\right\| \leq\|A X+X B\|,
$$

where $A, B, X$ are operators on a complex separable Hilbert space such that $A$ and $B$ are positive and $\||\cdot|||$ denotes a unitarily invariant norm.

The function $f(v)=\left\|A^{v} X B^{1-v}+A^{1-v} X B^{v}\right\|$ is convex on the interval $[0,1]$, attains its minimum at $v=\frac{1}{2}$, and attains its maximum at $v=0$ and $v=1$. Moreover, $f(v)=f(1-v)$ for $0 \leq v \leq 1$.

In [4], (1.1) is refined by Kittaneh by using the following equalities (see p.122 of [2]):

$$
f\left(\frac{a+b}{2}\right) \leq \frac{1}{b-a} \int_{a}^{b} f(t) d t \leq \frac{f(a)+f(b)}{2}
$$

where $f$ is a real-valued function which is convex on the interval $[a, b]$.

In [3], Feng used the following inequalities to get refinements of (1.1):

$$
f\left(\frac{a+b}{2}\right) \leq \frac{1}{b-a} \int_{a}^{b} f(t) d t \leq \frac{1}{4}\left(f(a)+2 f\left(\frac{a+b}{2}\right)+f(b)\right) \leq \frac{f(a)+f(b)}{2}
$$

where $f$ is a real-valued function which is convex on the interval $[a, b]$.

\section{黛 Springer}

(C) 2013 Wang; licensee Springer. This is an Open Access article distributed under the terms of the Creative Commons Attribution License (http://creativecommons.org/licenses/by/2.0), which permits unrestricted use, distribution, and reproduction in any medium, provided the original work is properly cited. 
In this paper, we prove that $\frac{1}{4}\left(f(a)+2 f\left(\frac{a+b}{2}\right)+f(b)\right)$ of $(1.3)$ can be replaced by $\frac{1}{32}(15 f(a)+$ $\left.2 f\left(\frac{a+b}{2}\right)+15 f(b)\right)$, and based on the inequalities

$$
\begin{aligned}
f\left(\frac{a+b}{2}\right) & \leq \frac{1}{b-a} \int_{a}^{b} f(t) d t \leq \frac{1}{32}\left(15 f(a)+2 f\left(\frac{a+b}{2}\right)+15 f(b)\right) \\
& \leq \frac{f(a)+f(b)}{2},
\end{aligned}
$$

we give some new refinements of Heinz inequalities of matrices.

Our results are better than the ones in [4], but we cannot say that our results are better than the ones in [3].

\section{Main results}

In [4], Kittaneh gave some refinements of the Heinz inequalities by using the previous lemma. In the following, we will use the following lemma to obtain several better refinements of the Heinz inequalities.

Lemma 1 Let $f$ be a real-valued function which is convex on the interval $[a, b]$. Then

$$
f\left(\frac{a+b}{2}\right) \leq \frac{1}{b-a} \int_{a}^{b} f(t) d t \leq \frac{1}{32}\left(15 f(a)+2 f\left(\frac{a+b}{2}\right)+15 f(b)\right) \leq \frac{f(a)+f(b)}{2} .
$$

Proof Since $f\left(\frac{a+b}{2}\right) \leq \frac{f(a)+f(b)}{2}$, we know that $15 f(a)+2 f\left(\frac{a+b}{2}\right)+15 f(b) \leq 16 f(a)+16 f(b)$.

Thus

$$
\frac{1}{32}\left(15 f(a)+2 f\left(\frac{a+b}{2}\right)+15 f(b)\right) \leq \frac{f(a)+f(b)}{2} .
$$

Next, we will prove the following inequality:

$$
\frac{1}{b-a} \int_{a}^{b} f(t) d t \leq \frac{1}{32}\left(15 f(a)+2 f\left(\frac{a+b}{2}\right)+15 f(b)\right) .
$$

We have

$$
\begin{aligned}
\frac{1}{b-a} \int_{a}^{b} f(t) d t & =\frac{1}{b-a}\left(\int_{a}^{\frac{a+b}{2}} f(t) d t+\int_{\frac{a+b}{2}}^{b} f(t) d t\right) \\
& \leq \frac{1}{b-a}\left(\frac{f(a)+f\left(\frac{a+b}{2}\right)}{2} \cdot \frac{b-a}{2}+\frac{f\left(\frac{a+b}{2}\right)+f(b)}{2} \cdot \frac{b-a}{2}\right) \\
& =\frac{1}{4}\left(f(a)+2 f\left(\frac{a+b}{2}\right)+f(b)\right) \\
& =\frac{1}{32}\left(8 f(a)+16 f\left(\frac{a+b}{2}\right)+8 f(b)\right) \\
& \leq \frac{1}{32}\left(8 f(a)+2 f\left(\frac{a+b}{2}\right)+7(f(a)+f(b))+8 f(b)\right) \\
& =\frac{1}{32}\left(15 f(a)+2 f\left(\frac{a+b}{2}\right)+15 f(b)\right) .
\end{aligned}
$$


Applying the previous lemma to the function $f(v)=\left\|A^{v} X B^{1-v}+A^{1-v} X B^{v}\right\| \mid$ on the interval $[\mu, 1-\mu]$ when $0 \leq \mu \leq \frac{1}{2}$, and on the interval $[1-\mu, \mu]$ when $\frac{1}{2} \leq \mu \leq 1$, we obtain refinement of the first inequality in (1.1).

Theorem 1 Let $A, B, X$ be operators such that $A, B$ are positive. Then, for $0 \leq \mu \leq 1$ and for every unitarily invariant norm, we have

$$
\begin{aligned}
2\left\|A^{\frac{1}{2}} X B^{\frac{1}{2}}\right\| & \leq \frac{1}{|1-2 \mu|}\left|\int_{\mu}^{1-\mu}\left\|A^{v} X B^{1-v}+A^{1-v} X B^{v}\right\| d v\right| \\
& \leq \frac{1}{16}\left(15\left\|A^{\mu} X B^{1-\mu}+A^{1-\mu} X B^{\mu}\right\|+2\left\|A^{\frac{1}{2}} X B^{\frac{1}{2}}\right\|\right) \\
& \leq\left\|A^{\mu} X B^{1-\mu}+A^{1-\mu} X B^{\mu}\right\| .
\end{aligned}
$$

Proof First assume that $0 \leq \mu \leq \frac{1}{2}$. Then it follows by the previous lemma that

$$
\begin{aligned}
f\left(\frac{1-\mu+\mu}{2}\right) & \leq \frac{1}{1-2 \mu} \int_{\mu}^{1-\mu} f(t) d t \\
& \leq \frac{1}{32}\left(15 f(\mu)+2 f\left(\frac{1-\mu+\mu}{2}\right)+15 f(1-\mu)\right) \\
& \leq \frac{f(\mu)+f(1-\mu)}{2},
\end{aligned}
$$

and so

$$
\begin{aligned}
f\left(\frac{1}{2}\right) & \leq \frac{1}{1-2 \mu} \int_{\mu}^{1-\mu} f(t) d t \\
& \leq \frac{1}{16}\left(15 f(\mu)+f\left(\frac{1}{2}\right)\right) \\
& \leq f(\mu) .
\end{aligned}
$$

Thus,

$$
\begin{aligned}
2\left\|A^{\frac{1}{2}} X B^{\frac{1}{2}}\right\| & \leq \frac{1}{1-2 \mu} \int_{\mu}^{1-\mu}\left\|A^{v} X B^{1-\nu}+A^{1-\nu} X B^{v}\right\| d v \\
& \leq \frac{1}{16}\left(15\left\|A^{\mu} X B^{1-\mu}+A^{1-\mu} X B^{\mu}\right\|+2\left\|A^{\frac{1}{2}} X B^{\frac{1}{2}}\right\|\right) \\
& \leq\left\|A^{\mu} X B^{1-\mu}+A^{1-\mu} X B^{\mu}\right\| .
\end{aligned}
$$

Now, assume that $\frac{1}{2} \leq \mu \leq 1$. Then, by applying (2.2) to $1-\mu$, it follows that

$$
\begin{aligned}
2\left\|A^{\frac{1}{2}} X B^{\frac{1}{2}}\right\| & \leq \frac{1}{2 \mu-1} \int_{1-\mu}^{\mu}\left\|A^{v} X B^{1-\nu}+A^{1-v} X B^{v}\right\| d v \\
& \leq \frac{1}{16}\left(15\left\|A^{\mu} X B^{1-\mu}+A^{1-\mu} X B^{\mu}\right\|+2\left\|A^{\frac{1}{2}} X B^{\frac{1}{2}}\right\|\right) \\
& \leq\left\|A^{\mu} X B^{1-\mu}+A^{1-\mu} X B^{\mu}\right\| .
\end{aligned}
$$


Since

$$
\begin{aligned}
& \lim _{\mu \rightarrow \frac{1}{2}} \frac{1}{|1-2 \mu|}\left|\int_{\mu}^{1-\mu}\right|\left\|A^{v} X B^{1-v}+A^{1-v} X B^{v}\right\| d v \mid \\
& \quad=\lim _{\mu \rightarrow \frac{1}{2}} \frac{1}{16}\left(15\left\|A^{\mu} X B^{1-\mu}+A^{1-\mu} X B^{\mu}\right\|+2\left\|A^{\frac{1}{2}} X B^{\frac{1}{2}}\right\|\right) \\
& \quad=2\left\|A^{\frac{1}{2}} X B^{\frac{1}{2}}\right\|,
\end{aligned}
$$

the inequalities in (2.1) follow by combining (2.2) and (2.3).

Applying the previous lemma to the function $f(v)=\left\|A^{v} X B^{1-v}+A^{1-v} X B^{v}\right\| \mid$ on the interval $\left[\mu, \frac{1}{2}\right]$ when $0 \leq \mu \leq \frac{1}{2}$, and on the interval $\left[\frac{1}{2}, \mu\right]$ when $\frac{1}{2} \leq \mu \leq 1$, we obtain the following.

Theorem 2 Let $A, B, X$ be operators such that $A, B$ are positive. Then, for $0 \leq \mu \leq 1$ and for every unitarily invariant norm, we have

$$
\begin{aligned}
&\left\|A^{\frac{2 \mu+1}{4}} X B^{\frac{3-2 \mu}{4}}+A^{\frac{3-2 \mu}{4}} X B^{\frac{2 \mu+1}{4}}\right\| \mid \\
& \leq \frac{2}{|1-2 \mu|}\left|\int _ { \mu } ^ { \frac { 1 } { 2 } } \left\|\left|A^{v} X B^{1-v}+A^{1-v} X B^{v} \| d v\right|\right.\right. \\
& \leq \frac{1}{32}\left(15\left\|A^{\mu} X B^{1-\mu}+A^{1-\mu} X B^{\mu}\right\|+2\left\|A^{\frac{2 \mu+1}{4}} X B^{\frac{3-2 \mu}{4}}+A^{\frac{3-2 \mu}{4}} X B^{\frac{2 \mu+1}{4}}\right\|\right. \\
&\left.+30\left\|A^{\frac{1}{2}} X B^{\frac{1}{2}}\right\|\right) \\
& \leq \frac{1}{2}\left(\left\|A^{\mu} X B^{1-\mu}+A^{1-\mu} X B^{\mu}\right\|+2\left\|A^{\frac{1}{2}} X B^{\frac{1}{2}}\right\|\right) .
\end{aligned}
$$

Inequality (2.4) and the first inequality in (1.1) yield the following refinement of the first inequality in (1.1).

Corollary 1 Let $A, B, X$ be operators such that $A, B$ are positive. Then, for $0 \leq \mu \leq 1$ and for every unitarily invariant norm, we have

$$
\begin{aligned}
& 2\left\|A^{\frac{1}{2}} X B^{\frac{1}{2}}\right\| \\
& \leq \\
& \leq|| A^{\frac{2 \mu+1}{4}} X B^{\frac{3-2 \mu}{4}}+A^{\frac{3-2 \mu}{4}} X B^{\frac{2 \mu+1}{4}} \| \\
& \leq \frac{2}{|1-2 \mu|}\left|\int_{\mu}^{\frac{1}{2}}\left\|A^{v} X B^{1-v}+A^{1-v} X B^{v}\right\| d v\right| \\
& \leq \frac{1}{32}\left(15\left\|A^{\mu} X B^{1-\mu}+A^{1-\mu} X B^{\mu}\right\|+2\left\|A^{\frac{2 \mu+1}{4}} X B^{\frac{3-2 \mu}{4}}+A^{\frac{3-2 \mu}{4}} X B^{\frac{2 \mu+1}{4}}\right\|\right. \\
&\left.+30\left\|A^{\frac{1}{2}} X B^{\frac{1}{2}}\right\|\right) \\
& \leq \frac{1}{2}\left(\left\|A^{\mu} X B^{1-\mu}+A^{1-\mu} X B^{\mu}\right\|+2\left\|A^{\frac{1}{2}} X B^{\frac{1}{2}}\right\|\right) \\
& \leq\left\|A^{\mu} X B^{1-\mu}+A^{1-\mu} X B^{\mu}\right\| .
\end{aligned}
$$

Applying the previous lemma to the function $f(v)=\left\|A^{v} X B^{1-v}+A^{1-v} X B^{v}\right\|$ on the interval $[0, \mu]$ when $0 \leq \mu \leq \frac{1}{2}$, and on the interval $[\mu, 1]$ when $\frac{1}{2} \leq \mu \leq 1$, we obtain the following theorem. 
Theorem 3 Let $A, B, X$ be operators such that $A, B$ are positive. Then,

(1) for $0 \leq \mu \leq \frac{1}{2}$ and for every unitarily norm,

$$
\begin{aligned}
& \left\|A^{\frac{\mu}{2}} X B^{1-\frac{\mu}{2}}+A^{1-\frac{\mu}{2}} X B^{\frac{\mu}{2}}\right\| \\
& \quad \leq \frac{1}{\mu} \int_{0}^{\mu}\left\|A^{v} X B^{1-v}+A^{1-v} X B^{v}\right\| d v \\
& \quad \leq \frac{1}{32}\left(15\|A X+X B\|+2\left\|A^{\frac{\mu}{2}} X B^{1-\frac{\mu}{2}}+A^{1-\frac{\mu}{2}} X B^{\frac{\mu}{2}}\right\|+15\left\|A^{\mu} X B^{1-\mu}+A^{1-\mu} X B^{\mu}\right\|\right) \\
& \quad \leq \frac{1}{2}\left(\|A X+X B\|+\left\|A^{\mu} X B^{1-\mu}+A^{1-\mu} X B^{\mu}\right\|\right)
\end{aligned}
$$

(2) for $\frac{1}{2} \leq \mu \leq 1$ and for every unitarily norm,

$$
\begin{aligned}
& \left\|A^{\frac{1+\mu}{2}} X B^{\frac{1-\mu}{2}}+A^{\frac{1-\mu}{2}} X B^{\frac{1+\mu}{2}}\right\| \\
& \quad \leq \frac{1}{1-\mu} \int_{\mu}^{1}\left\|A^{v} X B^{1-v}+A^{1-v} X B^{v}\right\| d v \\
& \leq \frac{1}{32}\left(15\|A X+X B\|+2\left\|A^{\frac{1+\mu}{2}} X B^{\frac{1-\mu}{2}}+A^{\frac{1-\mu}{2}} X B^{\frac{1+\mu}{2}}\right\|+15\left\|A^{\mu} X B^{1-\mu}+A^{1-\mu} X B^{\mu}\right\|\right) \\
& \quad \leq \frac{1}{2}\left(\|A X+X B\|+\left\|A^{\mu} X B^{1-\mu}+A^{1-\mu} X B^{\mu}\right\|\right) .
\end{aligned}
$$

Since the function $f(v)=\left\|A^{v} X B^{1-v}+A^{1-v} X B^{v}\right\| \mid$ is decreasing on the interval $\left[0, \frac{1}{2}\right]$ and increasing on the interval $\left[\frac{1}{2}, 1\right]$, and using the inequalities (2.6) and (2.7), we obtain the refinement of the second inequality in (1.1).

Corollary 2 Let $A, B, X$ be operators such that $A, B$ are positive. Then, for $0 \leq \mu \leq 1$ and for every unitarily invariant norm, we have

(1) for $0 \leq \mu \leq \frac{1}{2}$ and for every unitarily norm,

$$
\begin{aligned}
\| A^{\mu} & X B^{1-\mu}+A^{1-\mu} X B^{\mu} \| \\
& \leq\left\|A^{\frac{\mu}{2}} X B^{1-\frac{\mu}{2}}+A^{1-\frac{\mu}{2}} X B^{\frac{\mu}{2}}\right\| \\
& \leq \frac{1}{\mu} \int_{0}^{\mu}\left\|A^{v} X B^{1-v}+A^{1-v} X B^{v}\right\| d v \\
& \leq \frac{1}{32}\left(15\|A X+X B\|+2\left\|A^{\frac{\mu}{2}} X B^{1-\frac{\mu}{2}}+A^{1-\frac{\mu}{2}} X B^{\frac{\mu}{2}}\right\|+15\left\|A^{\mu} X B^{1-\mu}+A^{1-\mu} X B^{\mu}\right\|\right) \\
& \leq \frac{1}{2}\left(\|A X+X B\|+\left\|A^{\mu} X B^{1-\mu}+A^{1-\mu} X B^{\mu}\right\|\right) \\
& \leq\|A X+X B\| ;
\end{aligned}
$$

(2) for $\frac{1}{2} \leq \mu \leq 1$ and for every unitarily norm,

$$
\begin{aligned}
& \left\|A^{\mu} X B^{1-\mu}+A^{1-\mu} X B^{\mu}\right\| \\
& \quad \leq\left\|A^{\frac{1+\mu}{2}} X B^{\frac{1-\mu}{2}}+A^{\frac{1-\mu}{2}} X B^{\frac{1+\mu}{2}}\right\| \\
& \quad \leq \frac{1}{1-\mu} \int_{\mu}^{1}\left\|A^{\nu} X B^{1-v}+A^{1-v} X B^{\nu}\right\| d \nu
\end{aligned}
$$




$$
\begin{aligned}
& \leq \frac{1}{32}\left(15\|A X+X B\|+2\left\|A^{\frac{1+\mu}{2}} X B^{\frac{1-\mu}{2}}+A^{\frac{1-\mu}{2}} X B^{\frac{1+\mu}{2}}\right\|+15\left\|A^{\mu} X B^{1-\mu}+A^{1-\mu} X B^{\mu}\right\|\right) \\
& \leq \frac{1}{2}\left(\|A X+X B\|+\left\|A^{\mu} X B^{1-\mu}+A^{1-\mu} X B^{\mu}\right\|\right) \\
& \leq\|A X+X B\| .
\end{aligned}
$$

It should be noticed that in inequalities (2.6) to (2.9),

$$
\begin{aligned}
& \lim _{\mu \rightarrow 0} \frac{1}{\mu} \int_{0}^{\mu}\left\|A^{v} X B^{1-v}+A^{1-v} X B^{v}\right\| d v, \\
& \lim _{\mu \rightarrow 1} \frac{1}{1-\mu} \int_{\mu}^{1}\left\|A^{v} X B^{1-v}+A^{1-v} X B^{v}\right\| d v \\
& \quad=\|A X+X B\| .
\end{aligned}
$$

\section{Competing interests}

The author declares that they have no competing interests.

Received: 27 April 2013 Accepted: 21 August 2013 Published: 8 September 2013

\section{References}

1. Bhatia, R, Davis, C: More matrix forms of the arithmetic-geometric mean inequality. SIAM J. Matrix Anal. Appl. 14, 132-136 (1993)

2. Bullen, PS: A Dictionary of Inequalities. Pitman Monographs and Surveys in Pure and Applied Mathematics, vol. 97. Addison-Wesley, Reading (1998)

3. Feng, Y: Refinements of the Heinz inequalities. J. Inequal. Appl. 2012, 18 (2012). doi:10.1186/1029-242X-2012-18

4. Kittaneh, F: On the convexity of the Heinz means. Integral Equ. Oper. Theory 68, 519-527 (2010)

\section{Submit your manuscript to a SpringerOpen ${ }^{\ominus}$ journal and benefit from:}

- Convenient online submission

- Rigorous peer review

- Immediate publication on acceptance

- Open access: articles freely available online

- High visibility within the field

- Retaining the copyright to your article 